\title{
Deutsches Wortgut in rumänischen Pflanzennamen. Ein Bericht aus der rumänischen Wörterbuchpraxis
}

\author{
Radu Drăgulescu, Department für romanische Studien, \\ Fakultät für Philologie und Theaterwissenschaften, \\ „Lucian Blaga" Universität, Sibiu, Rumänien \\ (radu.dragulescu@ulbsibiu.ro)
}

\begin{abstract}
Zusammenfassung: Mit dem Vorführen vielfältiger Spracheinflüsse in volkstümlichen Pflanzennnamen nimmt folgender Beitrag eine wichtige, jedoch ungenügend erforschte Erscheinung - Sprachschöpfung als Ergebnis der Pflanzenbezeichnungen und Ausdruck volkstümlicher Prägung - in den Blick. Ein Phänomen, das, wie von E. Coşeriu betrachtet wird, nicht genug hervorgehoben wird (da der einzelne Sprecher Schöpfer der Sprache/Poesie wurde, wann immer er eine Blume nannte). Unserer Meinung nach ist ein botanisches Lexikon einer bestimmten Region, einmal bekannt, nicht nur ein Thesaurus, aber auch ein Dokument der ansässigen Bevölkerung und Orte der Vergangenheit. Die botanische Volksterminologie hat in erster Linie einen praktischen Wert, der die Elemente des Pflanzenreiches innerhalb der gegebenen natürlichen Herrschaft kennzeichnet, unterscheidet und kategorisiert. Sie hat aber auch eine hohe theoretische Bedeutung für Linguisten, sowohl betreffend die Etymonen, zu denen sie zurückschicken, als auch die metaphorischen Bedeutungen, die die Phytonymen meist haben. Der Beitrag fokussiert auf einen ausgewählten Bereich der rumänischen Speziallexikografie und bietet einen Einblick in die jüngste lexikografische Erfassung rumänischer Pflanzennamen. Da jedoch das deutsche Wortgut in den herkömmlichen usuellen Pflanzenbezeichnungen von der rumänischen Fachliteratur eher marginal berücksichtigt und deren Besonderheiten nur vereinzelt erfasst und beschrieben worden sind, greifen die Ausführungen ausschließlich entlehnte und/oder dem Rumänischen angepasste Pflanzenbezeichnungen deutscher Herkunft auf. Andere außersprachliche Entsprechungen, Verwechslungen, irrtümliche Zuordnungen, sonstige Pflanzenarten oder Bildungen mit den Begriffen german/nemţesc (,deutsch') oder săsesc (,sächsisch') u.a. wurden hier nicht berücksichtigt. In unsere Arbeit soll dieses reiche Inventar an Pflanzennamen am Beispiel des hier vorkommenden deutschen Wortguts vorgestellt und erläutert werden.
\end{abstract}

Stichwörter: LEXIKOGRAFISCHE PRAXIS, BEDEUTUNGSERKLÄRUNG, SPEZIALWÖRTERBUCH, PFLANZENBEZEICHNUNGEN, ENTLEHNUNGSPROZESS, LEHNPRÄGUNG, LINGUISTICHER EINFLUSS, DEUTSCH, RUMÄNISCH, SIEBENBÜRGERSÄCHSISCH

Abstract: German Vocabulary in Romanian Plant Names. A Report of Romanian Lexicographic Practice. This paper examines an important but insufficiently investigated phenomenon - lexical innovation as a result of plant naming and the expression of 
national customs and traditions - by showing various linguistic influences in common plant names. E. Coşeriu considers this phenomenon to be given insufficient importance by linguists, given that fact that individual speakers became creators of language/poetry whenever they named a plant. I am of the opinion that a botanical lexicon of a particular region, once known, is not only a thesaurus, but can also be regarded as a document of a resident population or place in the past. First and foremost, botanical folk terminology has a practical value in that it identifies, distinguishes and categorizes members of the plant kingdom within a given natural realm. It, however, also has great theoretical significance for linguists, both with regard to the etymons from which they are derived, and to the metaphorical meanings that the phytonyms usually have. The article focuses on a selected area of Romanian specialized lexicography and offers an insight into the most recent lexicographic recording of Romanian plant names. Since, however, the German vocabulary in the commonly used plant names has been rather marginalized in the Romanian specialized literature, and since its characteristics have only been recorded and described loosely, the explanations are based solely on borrowed and/or adapted Romanian plant names of German origin. Other extraneous correspondences, mix-ups, erroneous assignments, other plant species or formations with the terms German/nemţesc ('deutsch') or Transylvanian-Saxon/săsesc ('sächsisch') etc., were not considered here. In my paper this rich inventory of plant names will be presented and exemplified using the German vocabulary listed here.

Keywords: LEXICOGRAPHIC PRACTICE, MEANING EXPLANATION, SPECIALIZED DICTIONARY, PHYTONYMS, LOAN PROCESS, CALQUE, LINGUISTIC INFLUENCE, GERMAN, ROMANIAN, TRANSYLVANIAN SAXON IDIOM

\section{Vorbemerkungen}

Folgende Ausführungen gehen von der Erkenntnis aus, dass mit der Erfassung des volkstümlichen Pflanzeninventars verschiedener Gebiete Rumäniens Sprachschätze und wertvolle Sprachzeugnisse vergangener Sprachgemeinschaften bewahrt werden. Das empirisch erhobene Sprachmaterial, eine Fundgrube insbesondere für Linguisten ${ }^{1}$, verdeutlicht Eigenheiten, die der wissenschaftlichen Fachwelt und auch dem interessierten Nichtfachpublikum zugänglich gemacht werden müssen. Auch gibt es hier einen großen Bedarf an noch zu erbringenden Forschungen: z.B. Erforschung der Etyma und des metaphorischen Sprachgebrauchs, Erfassung der geografischen Verteilung usueller Pflanzennamen. Bei der Prägung der botanischen Terminologie waren Besonderheiten der Pflanzenwelt in der betreffenden Landschaft für die Sprechergemeinschaft ausschlaggebend.

Das hier vorgestellte Material geht auf meine Zusammenarbeit mit Constantin Drăgulescu anlässlich der Ausarbeitung eines erklärenden Wörterbuchs rumänischer Pflanzennamen Diç̧ionarul explicativ al fitonimelor româneşti (2010) zurück. Dieses Wörterbuch ist eine wertvolle Ergänzung des 1968 von Borza veröffentlichten Wörterbuchs der Pflanzennamen (Dicţionar etnobotanic). Es erfasst einige tausend Pflanzennamen ungarischer, sächsischer, deutscher, französischer, englischer, russischer, ukrainischer, serbischer, bulgarischer und 
türkischer Herkunft und auch 10906 rumänische Bezeichnungen für insgesamt 2095 Pflanzenarten. Mit der Veröffentlichung dieses Wörterbuchs und eines weiteren, 2014 erschienenen Wörterbuchs (Dicţionar de fitonime româneşti) dokumentiert Drăgulescu 21839 rumänische Pflanzennamen für 3227 heimische und exotische, auch gezüchtete Pflanzenarten und legt die bisher umfangreichste lexikografisch erfasste Belegsammlung vor. Darüber hinaus wurden weitere 3070 Pflanzengattungen und 612 Bezeichnungen für Pflanzenteile berücksichtigt. Somit liegt ein wertvolles Korpus rumänischer (autochthoner) Pflanzennamen vor, das über 26000 Stichwörter umfasst.

Fast die Hälfte der bisher aufgenommenen Pflanzenbezeichnungen ist auch im Wortschatz der rumänischen Gegenwartssprache auszumachen. Ihr Geltungsbereich lässt sich wie folgt umreißen: Etwa 7000 werden im alltäglichen Sprachgebrauch verwendet, ungefähr 1000 nur von Fachleuten (Botanisten, Agronomen, Fachleute für Wald- und Forstwirtschaft, Gartenbaufachleute, Apotheker oder Ärzte) und ca. 2200 sind landesweit üblich. Die überregionale Verbreitung dieses Inventars ist vorwiegend den Hirten und Kaufleuten zu verdanken. Eine wichtige Rolle spielten auch die Lehrbücher und populärwissenschaftlichen Abhandlungen zu Heilpflanzen oder zum Anbau von genießbaren Pflanzen oder Zierpflanzen.

\section{Geschichtlicher ${ }^{2}$ Abriss der Überlieferung rumänischer Phytonyme}

Die erste Erwähnung eines Pflanzennamens geht auf das 14. Jahrhundert zurück und ist im Toponym Cornăţel (1306) belegt. Weitere Belege sind das Anthroponym Bradel (1348), später auch das Toponym Gura Brădăţelului (1456) und das Phytonym brad (1490). Ebenfalls aus dem 14. Jahrhundert belegt sind die Toponyme Răchita (1360), Jaleşul (1385), Sălcioara (1391-1392), Aniniş (1392), Ceret oder Nucet (1400). Aus dem 15. Jahrhundert sind über 30 Siedlungsnamen (Oikonyme), Berg- und Gebirgsnamen (Oronyme) und Gewässernamen (Hydronyme) überliefert, die aus Pflanzennamen hervorgegangen sind (Drăgulescu 2010). Hinzu kommen weitere Anthroponyme wie Laur (1443), Cimbru (1462), Săcară (1479) bzw. Secară (1482, 1487), Dzârnă (1488), Ceapă (1492), Brânduşă (1494) oder Ghinda (1497) (Drăgulescu 2010).

Aus dem 16. Jahrhundert stammen u.a. die Toponyme Frăsinet de Dumbravă (1502, 1504), Curpinel (1512), Fântâna Teiul (1517), Mănăstirea Brusturi (1518), Măceşul und Pelinul (1520), Scoruş (1519), Călinet (1548), Jugastru (1555), Zmeuret (1567), Părul (1571) und das Anthroponym Mălai (1507, 1517), die generische Pilzbezeichnung bureţi (Pl. 1509), die Pflanzenbezeichnungen chimen (,Kümmel'), grâu (,Weizen'), mărar (,Dill'), măslin (,Olivenbaum'), mujdar (,Knoblauch'), pătrănjen (,Petersilie'), smochin (,Feigenbaum'), urzică (,Brennessel') (1551), călin (,Schneeballstrauch'; 1557), cer (,Zerreiche'; 1577), arciariu (arţar) (,Ahorn'; 1579), pepene (,Melone'; 1598). ${ }^{3}$ In dem aus dem 16. Jahrhundert stammenden Sprachdenkmal Palia de la Orăştie (1582), die erste Übersetzung ins Rumänische der ersten zwei Bücher des Alten Testaments, unter dem Einfluss der Reforma- 
tion entstanden, sind die Pflanzenbezeichnungen ceapă (,Zwiebel'), coliandru (sic!) (,Koriander'), dafin (,Lorbeer'), mesteacăn (,Birke), stejar (,Eiche') belegt.

In den bedeutendsten Sammlungen und Enzyklopädien des 17. Jahrhunderts sind fast 700 rumänische Pflanzenbezeichnungen überliefert worden. ${ }^{4}$ Hier muss auf das reichhaltige Werk Dictionarium Valachico-Latinum des Anonymus Caransebesiensis (ca. 1693-1700) verwiesen werden, das 240 Pflanzennamen vermerkt. Auch Corbeas Wörterbuch (ca. 1691-1697) umfasst ca. 500 rumänische Bezeichnungen und gehört damit zu den umfangreichsten Sammlungen. ${ }^{5}$ Vereinzelt erscheinen rumänische Phytonyme auch in den Schriften von Varlaam (z.B. agud ,Maulbeere'; horciţă 'Senf') und Dosoftei (z.B. alac ,Dinkel'; marulă ,Lattich'; migdal ,Mandelbaum') belegt.

Aus dem 18. Jahrhundert sind viele rumänische Bezeichnungen in Kochbüchern überliefert. ${ }^{6}$ Im Calendariu pe 112 ani scos din multe feluri de cărţi (,Der Kalender für 112 Jahre aus vielen Büchern hervorgegangen'), der 1785 in Iaşi erschien, werden für 120 Heilpflanzen rumänische Bezeichnungen verzeichnet, viele davon sind unter dem Einfluss russischer Pflanzenbezeichnungen entstanden. Das wichtigste Werk "lateinischer, ungarischer und rumänischer" Phytonyme hat J. Benkő 1783 veröffentlicht. Hier werden 612 rumänische Bezeichnungen für 429 Pflanzengattungen erfasst, wobei vorwiegend Dorfbewohner aus Siebenbürgen und der Walachei als Gewährspersonen herangezogen wurden. Dieses Werk diente Sigerus (1791), Neustädter (1795) oder Veszelski (1798) als Vorbild, sodass Ende des 18 Jahrhunderts 1500 rumänische Pflanzenbezeichnungen aus fast allen Gebieten Rumäniens (Siebenbürgen, Banat, Walachei und Oltenien) für ca. 1000 Pflanzengattungen belegt sind. ${ }^{7}$

$\mathrm{Ab}$ dem 19. Jahrhundert kann eine eingehendere Beschäftigung und Sammeltätigkeit registriert werden. So sind über 3600 Bezeichnungen für 1520 Gattungen dokumentiert. Gh. Şincai (ca. 1810) übernimmt 188 rumänische Phytonyme für 167 Gattungen, die Sigerus verzeichnete und J.C.G. Baumgarten (1816) veröffentlicht in seinem Werk zur Siebenbürgischen Flora 296 Bezeichnungen für 257 Pflanzenarten. Die Mehrzahl der Bezeichnungen, die nicht von Benkő übernommen worden sind, sind Lehnbildungen, die auf fachliche oder lateinische Pflanzenbegriffe zurückgehen. J. Leonhard (1818) übernimmt ebenfalls einige rumänische Phytonyme von Sigerus und erfasst aber auch einige neue. Bobb (1822-1823) vermerkt in seinem Werk 635 Phytonyme, die entweder in einigen Vorgängerwerken bereits angeführt worden sind oder Lehnbildungen darstellen.

Das Lexikon Lexiconul românesc, latinesc, unguresc, nemţesc, 1825 in Budapest erschienen, führt außer den Phytonymen aus dem Werk von Corbea zahlreiche Lehnbildungen nach lateinischen, ungarischen oder deutschen Bezeichnungen an, davon auch einige, die als autochthon gelten und die im Dicţionarul Borza bereits vermerkt wurden. ${ }^{8}$

In seinem Manuskript aus dem Jahr 1841 nimmt Szabó 180 Pflanzennamen aus der Moldau auf. Einige Jahre später ergänzt Fuss (1847) die von Sigerus und Baumgarten erfassten rumänischen Bezeichnungen. Diese Samm- 
lung umfasst insgesamt 462 Phytonyme für 399 Pflanzenarten. Cipariu (1847) nimmt in seinem Glossar Bezeichnungen auf, die bereits im Dicţionar Corbea oder im Lexiconul de la Buda und in den Arbeiten von Sigerus und Fuss belegt sind. Czihac und Suţu (1849) erfassen über 600 rumänische Phytonyme, viele davon einheimische, aus der Moldau stammende Bezeichnungen und solche, die auf Lehnbildung zurückgehen. Bariț (1858-1859) hat ausgehend von Vorgängerwerken ebenfalls eine Liste mit 976 Bezeichnungen für 733 Pflanzenarten zusammengestellt und Sava Popovici-Barcianu (1868) ergänzt dessen Liste mit selbst gesammelten Bezeichnungen, sodass die Anzahl bekannter rumänischer Pflanzennamen 1065 beträgt. Das Verzeichnis von Fătu (1870) umfasst wenige einheimische Bezeichnungen, während Baronzi (1872) rumänische und französische Pflanzenarten, darunter Bezeichnungen für Obstbäume und Weinreben, anführt. Einen wichtigen Beitrag zur Erfassung einheimischer rumänischer Pflanzennamen aus der Region Caraş-Severin leistet Mangiuca (1874). Er kritisiert dabei die Autoren, die Pflanzenverzeichnisse veröffentlicht haben und nicht selbst als Sammler tätig waren. Diese haben sich vorwiegend auf Übersetzungen aus dem Griechischen, Lateinischen oder Deutschen beschränkt. Brândză $(1879-1883,1882)$ hat die volkstümlichen Bezeichnungen mit mehreren Hunderten Lehnbezeichnungen ergänzt und rumänische Bezeichnungen durch Lehnübersetzung griechischer oder lateinischer Fachberiffe geprägt. Auch Marian (1881-1908) und Hlibowski (1890) verzeichnen Hunderte von einheimischen Bezeichnungen aus der Bukowina. Porcius (18801882) ergänzt die Liste der Pflanzenbegriffe aus dem Lexiconul de la Buda mit denen von Bariţ şi Baumgarten. Er verzeichnet jedoch auch viele aus Năsăud gesammelte Bezeichnungen oder Eigenbildungen. Gleichfalls hat Crăiniceanu (1889, 1892-1893) autochthone Bezeichnungen und Lehnbegriffe aufgenommen, aber auch einige Namen, die auf Verwechslungen beruhen. ${ }^{9}$

Ende des vorigen Jahrhunderts sind bereits über 15000 rumänische Pflanzennamen für 2800 Arten belegt.10 Der Botanist Z.C. Panţu hat die Informationen aus dem Lexiconul de la Buda und die von Baumgarten, Fuss, Czihac/Suţu, Bariţiu, Brândză, Porcius u.a. zusammengetragenen und mit selbst gesammelten Bezeichnungen aus dem dörflichen und urbanen Umfeld ergänzt. Daher galt sein 1929 veröffentlichtes Nachschlagewerk, das 4650 rumänische Pflanzenbezeichnungen für fast 2000 Pflanzenarten erfasst, bis zum Erscheinen des Wörterbuchs von Al. Borza (1968; Dicţionarul etnobotanic) als das umfangreichste.

\section{Wandernde Namen. Autochthones und fremdes Wortgut in rumänischen Pflanzennamen}

In der rumänischen Ethnobotanik sind die auf Fachbegriffe oder auf fremde volkstümliche Bezeichnungen beruhenden (Lehn-)Bildungen unterrepräsentiert. Annähernd $80 \%$ rumänischer Pflanzennamen sind einheimische Bezeichnungen, die Mehrzahl davon sind Eigenbildungen der Dorfbewohner. Weitere 
$20 \%$ sind bildungssprachlich von Fachleuten (Botanisten, Apotheker, Ärzte, Philologen, Volkskundler, Kaufleute u.a.) geprägt worden. Diese sind z.B. im Deutschen, Französischen oder Englischen weitaus zahlreicher. Originelle rumänische Pflanzennamen belegen das kreative Potenzial und verdeutlichen Eigenheiten der benannten Pflanzen. Über 300 Bezeichnungen haben ihren ursprünglichen Geltungsbereich nicht verlassen, viele sind als Bildungen von Gelehrten oder Lehnbildungen sowie Okkasionalismen auszuweisen. Weitere 1500 Bezeichnungen haben Al. Borza und C. Drăgulescu als künstliche Bildungen, Übersetzungen oder Anpassungen von Fachbezeichnungen gewertet und in ihren Werken ausgeschlossen. ${ }^{11}$ Das Wörterbuch von Drăgulescu ignoriert auch 301 rumänische Phytonyme, die irrtümlicherweise bestimmten Pflanzenarten zugeordnet wurden sowie weitere ca. 300 Bezeichnungen, die in den Vorgängerwerken beim Abschreiben schriftlich falsch erfasst worden sind. Die häufigsten falsch transkribierten Phytonyme verzeichnet die Arbeit von Pârvu, Universul plantelor (1991) (Drăgulescu 2010).

Mit der Einstellung der Vermarktung einiger Heilpflanzen und Gewürze oder der Pflanzen für die Herstellung von Tinkturen sind über hundert Phytonyme aus dem mündlichen Sprachgebrauch verschwunden. Allerdings haben über 500 Bezeichnungen - insbesondere für Zierpflanzen oder zum Verzehr vorwiegend im 20. Jahrhundert Eingang ins Rumänische gefunden und gelten als weit verbreitet.

Die Wandlungen in der Namensgebung sind durch vielfältige Faktoren beeinflusst, da naturgegebene, geografische, klimatische oder pedologische Veränderungen auch Transformationen in der Zusammensetzung der Flora bedingen. Auch in der Ernährungsweise der Bevölkerung sind tiefgreifende Veränderungen auszumachen. Gleichfalls vermögen wirschaftliche, soziale, historische, kulturelle und nicht zuletzt sprachliche Entwicklungen Veränderungen in der Prägung von Pflanzennamen herbeizuführen. Die über 100 Gemüse- und Obstarten, die nicht in Rumänien angebaut oder noch vor zwei Jahhunderten nicht importiert wurden oder die neuen Pflanzen, die Gärten und Parks schmücken, erweckten die Phantasie vieler Bewunderer, die ihnen treffende Namen gaben. ${ }^{12}$ Andererseits wurden bestimmte traditionelle Pflanzen nicht mehr angebaut oder verwertet wie z.B. Hirse, Dinkel, Buchweizen, bestimmte Apfel-, Birnen-, Pflaumen-, Weizen- oder Rebarten u.a. ${ }^{13}$

Außer den einheimischen Bezeichnungen verzeichnet das rumänische Inventar an Phytonymen auch Übernahmen aus den Sprachen de Nachbarländer oder der in Rumänien ansässigen Völkergruppen, vorwiegend Ungarn, Sachsen, Serben, Bulgaren, Ukrainer, Russen, Türken. Bildungssprachliche Bezeichnungen gehen vorwiegend auf die Gelehrten zurück.

Die fremden Wörter und Pflanzenbezeichnungen - auch innerhalb ein und derselben Sprache - wurden bedauerlicherweise unterschiedlich schriftlich erfasst. Vorwiegend ungarische und deutsche Autoren verzeichneten rumänische Pflanzenbezeichnungen in einer ihrer Sprache üblichen Form, manchmal genau - z.B. znyeure, gyiotséj, jitze de jie, maku csari, frunsze de tsints 
desitye, juáre, heráne für znierură, ghiocei, iiţă de iie, macu ciorii, frunză de cinci deştie, iuoară, hărană oder Brendusch, Kretzischor, Tschireschelle für brânduşi, crețişor, cireşele - oder falsch (z.B. szkletsi mits anstatt scălci mici, ferukutze für fereguţă/feriguţă). Mitunter können die verzeichneten Pflanzennamen verschiedenartig gelesen werden. So z.B. flóre szóruluj für floarea soarelui und auch floarea sorului, stedse für şteje/ştegie ; szásztyiu erscheint als sastiu und sachiu, rujen wurde als ruien und rujen transkribiert, selnike wurde als şâlnică-şălnică gedeutet. Sâlnică, szimszene, Szinsiänie erscheinen in rumänischer Graphie mit i, anstatt sâmzene, sânzienie. Es sind auch Fälle auszumachen, wo die rumänische Schreibvariante nicht rekonstruiert werden konnte: z.B. jijetz mere für Pulsatilla, virczid'sa für Polypodium vulgare. Oft begegnen Inkongruenzen oder Auslassungen des bestimmten Artikels (des Genitiv-i). Z.B. lilie vunet (anstatt lilie vânătă), jarbe kodruluj (iarba codrului), lapte kinyeluj (laptele câinelui), puine babe (pâinea babii).

Manche rumänische Pflanzenbezeichnungen lassen sich indirekt auf einen indoeuropäischen Ursprung zurückführen. Viele entstammen dem Sprachgebrauch der in Rumänien lebenden Minderheiten (Ungarn, Sachsen, Roma) oder lassen sich als Ergebnis von Rückentlehnungsprozessen ausweisen. Etliche rumänische Bezeichnungen unterschiedlicher Pflanzen sind untergegangen und leben nur noch im Sprachgebrauch der Ungarn, Sachsen oder Roma fort. Manche Übernahmen aus dem Wortschatz der Minderheiten auf dem Gebiet Rumäniens zeigen Veränderungen in der Bedeutung, Lautung und Schreibweise.

Bei der Herleitung der Herkunft von Pflanzennamen haben die Lexikografen vergleichbare Bezeichnungen aus dem Sprachgebrauch der Minderheiten herangezogen und als direkte Übernahmen ausgewiesen, wobei oft unberücksichtigt blieb, dass der betreffende Name seinerseits von diesen Sprachen aus dem Rumänischen entlehnt wurde. Auch Pflanzenbezeichnungen aus anderen Sprachen sind berücksichtigt worden.

Die im Wörterbuch kodifizierten Herkunftsangaben verweisen vielfach auch auf außersprachliche Faktoren. Sie bieten Hinweise zu Formen und Farben einiger Pflanzenteile, zu ihrem Umfeld, zu Vorgängen und Lebenszyklen, zu ihrem Gebrauch und der mit ihnen verbundenen Bräuche, Legenden, rituellen Handlungen, mehr noch, die Pflanzenbenennungen sind von Fachbezeichnungen begleitet, da diese oft eine wichtige Rolle spielen und bei der Unterscheidung der Pflanzenarten mit gleichen Phytonymen hilfreich sind.

Die in den rumänischen Pflanzennamen belegten Präpositionen, Konjunktionen, Adverbien und Pronomina sind (vor)lateinischen Ursprungs: Präp. de (,von') < lat. de; la (,nach'; ,von') vgl. lat. illac oder aus der Präp. a "la" (,zu') aus der lat. ad, i.-e. Wurzel ${ }^{*}$ ad; $c u\left(, \mathrm{mit}^{\prime}\right)<$ lat. cum, i.-e. Wurzel ${ }^{*} \mathrm{ku}$; sub (,unter') < lat. sub, subtus; in (,in') < lat. in; din aus der Zusammensetzung de+in; pe (,auf') < lat. per, i.-e. Wurzel *per; Konj. vgl. lat. sic oder vorlateinisch; Adv. ca (,wie') < lat. quam, altind. ca; Pron. care (,welche, welcher, welches') vgl. lat. qualis ; se (,sich') < lat. se, i.-e. Wurzel *se). 
Im Folgenden soll dieses reiche Inventar an Pflanzennamen exemplarisch am Beispiel des hier vorkommenden deutschen Wortguts vorgestellt und erläutert werden. Es handelt sich hierbei um entlehnte und/oder dem Rumänischen angepasste Pflanzenbezeichnungen deutscher Herkunft. Andere außersprachliche Entsprechungen, Verwechslungen, irrtümliche Zuordnungen, sonstige Pflanzenarten oder Bildungen mit den Begriffen german/nemţesc (,deutsch') oder săsesc (,sächsisch') u.a. wurden hier nicht berücksichtigt.

Das reiche Lehngut lässt sich wie folgt gliedern:

(1) Direkte phonetisch unveränderte Übernahmen: acaţie (Robinia pseudacacia) < dt. Akazie; bambus (Phyllostachys viridiglaucens, Sasa palmata) $<\mathrm{dt}$. Bambus (franz. bambou < malayez. bambu); carfiol (Brassica oleracea var. botrys) < dt. Karfiol; chifăr, chifer (Pinus silvestris) < dt. Kiefer; Diptam (Dictamnus albus) < dt. Diptam; dost (Origanum vulgare) < sächs/dt. Dost; edălvais (Leontopodium alpinum) $<\mathrm{dt}$. Edelweiss; gerbera $($ Gerbera hybrida $)<\mathrm{dt}$. Gerbera (Fachbegriff/zur Ehrung des deutschen Botanikers Traugott Gerber); inghe (Callistephus chinensis) < dt./sächs. Inge (Antoponym); lilie (Iris florentina, Iris germanica, Lilium bulbiferum, Lilium candidum, Lilium martagon) $<\mathrm{dt}$. Lilie; mangold (Beta vulgaris var. cicla) $<\mathrm{dt}$. Mangold; pizang (Musa spp.) < dt. Pisang "Bananenpflanze"; ramie (Boehmeria nivea) $<\mathrm{dt}$. Ramie; vasărlilie (Hosta plantaginea) < dt. reg. Wasserlilie; vermut (Artemisia absinthium) Wermut; zegras (Zostera marina) $<\mathrm{dt}$. Seegras.

(2) Phonetisch veränderte Übernahmen aus der deutschen Sprache: acacie (Robinia pseudacacia) < dt. Akazie; bacolţi (Jungbirke der Betula pendula) < dt. Bachholz; bonă (Vicia faba) < dt. Bohne; borda miresii (Campanula fenestrella) < bartă, bortă, beartă, bertă (,Hauptband, Hauptschmuck, Blumenkranz') < dt. Barten, Berthe; Brinolă (Mentha pulegium) < dt. Brunelle; bufă (Quercus spp.); bufoi, bufon(iu) (Fagus silvatica) < dt. Buche (Fagus); Bedeutungserweiterung; gegenwärtig für ein beliebiges Gebüsch oder als Bezeichnung für einen jungen Wald (im Banat); buhaci, buhaş (Jungexemplare' von Abies alba, Fagus silvatica, Picea abies), buhăşer (Picea abies; ,Jungexemplar') < dt. Buche (Fagus); cartifiol, cartofiol, cartofiom (Brassica oleracea var. botrys) < dt. Karfiol; cartoafe (Dahlia cultorum, Solanum tuberosum) $<\mathrm{dt}$. Kartoffel; castan (Aesculus hyppocastanum, Castanea sativa) < dt. Kastanienbaum, wissenschaftl. Benennung castanea; cherbel, cherbăl (Chaerophyllum aureum); chervăl (Anthriscus cerefolium) < dt. Kälber(kropf), Kerbel bzw. Wald-/Wiesen-Kerbel < cerefolium < griech. chairophyllon "angenehmes Blatt"; cocarde (Gaillardia picta) < dt. Kokardenblume; creţăruş(i) (Bellis perennis) < cre(i)ţar, crăiţar < dt. Kreuzer (,Münze'), ung. krajcár; crumciri, crumpe, crumpei, crumpei de lună (Solanum tuberosum), crumpene (Helianthus tuberosus, Solanum tuberosum), crumpeni, crumperi, crumpi, crumpiri, crumple, crumpli, crupre (Solanum tuberosum) < dt. Grundbirne, dt. dial. krompire, krumbiir, krumpa; crumpei (Elaphomyces spp.) ein knolliger Pilz, der so benannt wurde, weil er wie eine Grundbirne aussieht; cule (Solanum 
tuberosum) Pflanzenname aus Kreis Hunedoara, eine Nachbildung nach dt. Knolle, Knulle, Kulen; cuţcrişoară, cuţcrişor (Pulmonaria officinalis) < dt. Kuhkreuz; enţură, enzură (Gentiana spp.) < inţură, ghinţură unter dem Einfluss des dt. Enzian; eră (Triticum aestivum) Name des Weizen, mit dem dt. Ähre und griech. er, ear "Frühling” zu vergleichen; erdăpane (Solanum tuberosum) < dt. Erdapfel; fefărminț (Mentha piperita) < dt. Pfefferminze; fenicel (Foeniculum vulgare) wahrscheinlich unter dem Einfluss des sächs. fienchəl, dt. Fenchel; fetică (Ficaria verna heute Ranunculus ficaria, Valerianella locusta) ${ }^{14}$; fiache (Viola odorata) vgl. dt. Veilchen, russ., slow. fialka; fidireie (Solanum tuberosum) zu vergleichen mit dem dt. Feldeier, Erdeier; finc(h)en, finchăn, fenhiel, finhien, finchil, finc(h)in, fincăl (Foeniculum vulgare) < sächs. fin(t)chəl, fienchəl, dt. Fenchel; flos (Linum usitatissimum) < dt. Flachs; garva, garvă (Achillea millefolium) < dt. Schafgarbe, Garbe, sächs. guar, guərblâm, altdt. garwe, garwa; ghinţiure (Gentiana punctata), ghinţură (Centaurium erythraea, Euphrasia stricta, Gentiana spp.), ghinţurea (Centaurium erythraea, Gentiana cruciata), ghinţurele (Gentiana clusii), ghințurică (Gentiana frigida, Gentiana lutea, Gentiana nivalis) wahrscheinlich aus jinţură/inţură/enţură (vgl. dt. Gentian und Enzian und die wissenschaftliche Bezeichnung Gentiana nach dem Namen des illyrischen Königs Gentius); gonele (Solanum tuberosum) aus dem dt. Knolle, oder *gogonele (,unreife Tomaten'); grap (Rubia trinctorum) < dt. Krapp; hăidănuş(ă) (Viburnum opulus); besonders die Früchte < dt. Heidenkirsch(en); hulcă (Triticum aestivum var. muticum) möglich vom dt. Hülle, Hülse; inţură (Gentiana spp.), inţurea (Gentiana spp.), inţurică (Gentiana lutea) < rum. ghinţură; levcoaie (Cheiranthus cheiri) < dt. Levkoje; libistoc (Levisticum officinale) < dt. Liebstock; liză (Pelargonium zonale) < dt. Liese (Anthroponym); maieran, maioran, maiorean, măierean $(\breve{a})$, maiorană, măgheran (Majorana hortensis, Origanum vulgare), măgherean (Majorana hortensis), măghieran, măghiran, măghieran, măgieran, măgiran (Majorana hortensis), măioreană (Majorana hortensis): < dt./sächs. Majoran, Meieran; minuţe (Bellis perennis) < dt. Münze (Gänseblümchen); mutmel, mutruel, muturel (Triticum dicoccum): vgl. dt. dial. Mudelweissa (Triticum aestivum var. muticum) (Zweikorn) bzw. rum. mudmel, mutmel (,feines Weizenmehl') < dt. Mudmehl; nudli (Portulaca grandiflora) < dt. Nudel 15; ortanţă, ortensă, ortensie, ortenzie (Hydrangea opuloides) < wissenschaftl. Hortensia opuloides, $\mathrm{dt}$. Hortensie, fr. hortensia; petersil (Petroselinum hortense) $<\mathrm{dt}$. Petersil(ie); pilzene (Boletus edulis) < dt. Pilz, Pilze; pomaranţă, pomeranţă (Citrus aurantiaca) < dt. Pomeranze (> russ. pomeraneţ); pospanc, pospang, puspan, puşpan, sospa(i)n (Buxus sempervirens) < dt. Buchsbaum > ung. puspáng, puszpáng; rapăn (Capsella bursa-pastoris) < dt. Rappe, Rappen (,Münze'), da die Früchte so aussehen; râpac (Brassica napus, Brassica rapa), râpă, râpe (Beta vulgaris, Solanum tuberosum), rips (Brassica napus) < dt./sächs. Rübe, R(i)ep (Beta vulgaris) vgl. dt. Raps, Reps(kohl), ukr. rîpa (Brassica rapa), slaw. rěpa ,Speiserübe'; rebizlă, ribiză, ribizil, ribizle, ribizli, ribizlă, ribuţe, răbizăa, ribizle (Ribes rubrum) $<$ dt. Ribisel, landl. Ribiseln $(<$ Ribes $<$ arab. ribās), davon 
auch die ung. ribizli, ribizke, slovak. ribezl'a; rosmailin (Rosmarinus officinalis), rosmalin (Artemisia abrotanum, Rosmarinus officinalis), rosmarin (Artemisia abrotanum, Artemisia annua, Rosmarinus officinalis), rosmarint, rosmălin, rosmărin, rosmolin (Rosmarinus officinalis), rozmalin (Artemisia abrotanum, Rosmarinus officinalis), rozmarin (Artemisia abrotanum, Asparagus officinalis, Asparagus sprengeri, Daphne cneorum, Rosmarinus officinalis), rozmolin (Rosmarinus officinalis) aus dt. Rosmarin, it. rosmarino, davon auch ung. rosmarin $(\mathfrak{t})$, russ. rozmarin, möglicherweise auch aus der wissenschaftlichen Benennung Rosmarinus < griech. rhops (,kleiner Strauch'; griech. myrinos ,riechbar, geruchsvoll, balsamisch' oder lat. ros ,Tau' und lat. marinus, des Meeres'; ruben (Beta vulgaris) < dt. Rübe, Pl. Rüben; silairei (Apium graveolens) < dt. Sellerie; simsă (Juncus effusus, Juncus inflexus) < dt. Simse; soloagăr (Helianthus annuus) wahrscheinlich aus dt. Sonn(e)augen, auf die Blüten hinweisend; spichinat, spichinel (Lavandula angustifolia şi spp.) dt. Spickenard, Spicke; Lavandula spica < lat. spicum "Ähre”, auf die Blüten hinweisend; spinac (Spinacia oleracea) < Kontamination zwischen rum. spanac und $\mathrm{dt}$. Spinat; spinat (Spinacia oleracea, Atriplex hortensis) < dt. Spinat, sächs. špinât (Spinacia oleracea); streliţia (Strelitzia sp.) < dt. Strelitzie bzw. wissenschaftliche Benennung16; şalotă (Allium ascalonicum) < dt. Schalotte(n), fr. echalote, engl. Shallot; şlaierblume (Gypsophila paniculata) < dt. Schleierblume $>$ skr. šlajer (trava); şpic (Lavandula angustifolia) < dt. Spicke < Lavandula spica; ştainglezăr (Melilotus albus, Melilotus officinalis) vgl. dt. Steinklee; titenchi, titenghi (Coprinus atramentarius) das rum. Phytonym könnte eine Verzerrung der deutschen Bezeichnung sein oder vom i.-e. Wurzel *teng(,weichmachen, feuchtmachen') abstammen; toibă (Bromus secalinus) $<\mathrm{dt}$. Taubhafer; triftărele (Clitocybe infundibuliformis, Craterellus cornucopioides, Petunia hybrida): die ersten zwei Arten bezeichnen trichterförmige Pilze $(<$ dt. Trichter), die letzte ist eine Zierpflanze mit einer trichterförmigen Blumenkrone; trimpăn, trinpăn (Sedum acre) scheint eine Kontamination zwischen der deutschen Benennung Tripmadam und die rum. trânjen, trânjin zu sein; ţâncraus (Equisetum arvense) < dt. Zinnkraut; ţâtroană (Citrus limon, Philadelphus coronarius), țâtron (Hedera helix, Philadelphus coronarius, Ruscus hypoglossum, Viscum album), ţitroană, ţitrom (Citrus limon), țitron (Citrus limon, Philadelphus coronarius) $<\mathrm{dt}$. Zitrone, Zitron und ung. citrom, citron (Citrus limon) < dt. Zitrone, Zitron (Citrus limon), im Süden Siebenbürgens bezeichnet zitron eine immergrüne Pflanze, die Blumen der Philadelphus coronarius haben einen zitronenähnlichen Geruch; ţicorie (Cichorium intybus $)<\mathrm{dt}$. Zichorie > ung. cikoria; ţimat, ţimărt, ţimăt, ţimet, ţâmărt (Cynnamomum zeylanicum) < dt. Zimmet; ţucroş, ţucuran, ţucurechi (Beta vulgaris var. altissima) < dt. Zuckerrübe, sächs. zackerriep, ung. cukorrépa, cukrosrépa.

(3) Übernahmen aus dem siebenbürgisch-sächsischen Dialekt: apricops, apricos, apricoz, apricoază, abricoji, abricoz (Armeniaca vulgaris; heute: Prunus armeniaca) sächs. Aprikôs < dt. Aprikose; banat, bănat vgl. sächs. banat, 
bənat, bonât, banötek, banədick, bənâti(k), dt. Bonat, Banat, Benat, Bonot < dt. Benedikt oder i.-e. *man- ( $>$ *ban-) (,gut'), die Pflanze riecht sehr angenehm; berten, bertin (Artemisia dracunculus) < sächs. biërtrem, dt. Bertram; bonă (Vicia faba) aus rum. boană, bob, boabă < sächs. bon, bun, dt. Bohne; boreţ (Borago officinalis) < sächs. boretsch, serb. porec, poreč, skr. borač, $\mathrm{dt}$. Boretsch, Burets, ung. burecs, aus dem lat. *boletis, -em (anstatt boletus, nach dem griech. $\beta \omega \lambda ı \tau n)$; calarabă, calarambă, călărabe, caralabă, cararabe, cărălabă (Brassica oleracea var. gongyloides), chelărabe (Brassica oleracea var. acephala), chelarabă, chelărabe vinete, chelărabi, chelărabie (Brassica oleracea var. gongyloides), chelărade (Brassica oleracea var. acephala, Brassica oleracea var. gongyloides), chelerab (Brassica oleracea var. gongyloides) < sächs. kalərabən, kalərâbi, dt. Kohlrabi (> ung. kalaráb, karalábé, skr. keleraba, poln. kalarepa u.a.); cârneauă, hernave (Hypericum perforatum): vgl. sächs. kirnjan, harnäo, harnâ, harnau und dt. dial. Karnoul, Hartenau, Hertenau; chel, chil, chiel, chil (Brassica oleracea var. sabauda) sächs. ki(e)l, kîl, dt. Köhl(kraut) > skr. kelj, ung. kelkáposzta; cherbel, cherbăl (Chaerophyllum aureum), chervăl (Anthriscus cerefolium) < dt. Kälber(kropf), dt. Kerbel, sächs. kiərwəl, kerwəl, Wald-/Wiesen-Kerbel, < cerefolium < griech. chairophyllon (, angenehmes Blatt'), davon auch fr. cerfeuil; clocăboambă (Aquilegia vulgaris) < sächs. kliokəbläom, klokəblommən, dt. Glocke(n)blume; cricin, crihim, crihin, crichin (Prunus insititia) < sächs. kriehen, krächən; crumciri, crumpe, crumpei, crumpene (Helianthus tuberosus, Solanum tuberosum), crumpeni, crumperi, crumpi, crumpiri, crumple, crumpli, crupre (Solanum tuberosum) vgl. skr. krumpir, ung. krumpli < sächs. krumpirn, dt. Grundbirne, dt. reg. krompire, krumbiir, krumpa beeinflusst auch von ung. kolompér, kolompéra, krompé, krumpi, krumpli < dt. Grundbirne, an manchen Orten bedeuten diese Namen „krumme Knollen” (in Anlehnung an dt. krumm; Krummbirne); dost (Origanum vulgare) vgl. sächs. Dost; fenicel (Foeniculum vulgare) aus der wissenschaftlichen Benennung, lat. foeniculum, wahrscheinlich vom sächs. fienchəl, dt. Fenchel beeinflusst; finc(h)en, finchăn, fenhiel, finhien, finchil, finc(h)in, fincăl (Foeniculum vulgare) < sächs. fin(t)chəl, fienchəl; foalcer (Mathiola incana), foalchine, foalâne, foaline (Mathiola incana), foaltăr, foalter (Mathiola incana), foaltine (Cheiranthus cheiri, Mathiola incana), foahine (Tropaeolum majus), foanchine (Tagetes erecta) < sächs. foaltchər, vâltchər, foalen < dt. Veilchen (Mathiola incana), săchs. giel foalen, dt. gelbes Veilchen (Cheiranthus cheiri), sächs. voâlchen, dt. Veilchen (Viola spp.); garva, garvă (Achillea millefolium) < sächs. guar, guərblâm < dt. Schafgarbe, Garbe, altdt. garwe, garwa (,Garbe, Bündel'); grubă, grube, grumbă(i), grumbănă, grumbe, grumcizi, grumpă, grumpe, grumpene, grumpeni, grumpi, grumpin(i) (Solanum tuberosum) < sächs. grumpir(rə)n, grumpîr, grompîr, dt. Grundbirne, Grunbir, Gromper; heber (Lagenaria siceraria) < sächs. Heber/hebər; heciumpeci, heciunpeci (die Früchte und die Konfitüre von Rosa canina) < sächs. hätschenpätsch, hetschumpetsch; holăr (Sambucus nigra) < sächs./dt. Holler; inghe, inguţe (Callistephus chinensis) < sächs./dt. Inge (Anthroponym); liurbă(u), l(i)urben, l(i)urber, liorbe, (Laurus nobilis) < 
sächs. lührber(buhm), lu(i)rbən, lu(i)rbər, liurbər, dt. Lorbeer; liurben, lurbău (Syringa vulgaris) < sächs. lorbeer, lorbeerbaum (Syringa vulgaris); liză (Pelargonium zonale) < germ/sächs. Liese, liurbă(u), l(i)urben, l(i)urber, liorbe (Laurus nobilis) < sächs. lührber(buhm), lu(i)rbən, lu(i)rbər, liurbər, dt. Lorbeer; liurben, lurbău (Syringa vulgaris) < sächs. lorbeer, lorbeerbaum (Syringa vulgaris); mure (Daucus carota) < sächs. murr, murrən, dt. Möhre, slaw. murky; părădaice, părădaisăne, părădaise, părădăi, părădaişi, părădazine, părădăici, parad(a)ise, paradaice, paradaise, paradice, paradici, bărădăi, porobici, porodăi, porodăici, porodeici, porodică, porodici, porodiciuri, porodiţă (Lycopersicon esculentum) < sächs. Parədeis, dt. Paradeis(apfel), Paradies(apfel), ung. paradicsom < dt. Paradeisen, unter den rumänischen Pflanzenbenennungen gibt es Mischbildungen zwischen sächs. parədeis und ung. paradicsom, manchmal auch serb. paradajz, mit der Grundform vom griech. parádeisos (,umschlossenes Gebiet reserviert für den König Persiens') abstammend 17 ; rabarbară (Rheum officinale), rabarbură (Rheum officinale, Rheum palmatum), rabarbăr (Rheum rhabarbarum, Rheum rhaponticum), rebarbar( $\breve{\text { ) }}$ (Rheum officinale, Rheum palmatum, Rheum rhaponticum), rebarboră, robarbă, robarbăr (Rheum palmatum) < sächs. rabarbər, dt. R(h)abarber, it. rabarbaro < rhabarbarum, radix barbarum "Barbarenwurzel”; spargă (Asparagus officinalis) < sächs. spargəl, dt. Spargel > ung. spárga; şarlai (Salvia sclarea), şerlai (Salvia aethiopis, Salvia sclarea), şerlau (Salvia aethiopis) < sächs. schârlôch, dt. Scharlachkraut (Salvia sclarea); şnirling (Allium schoenoprasum) < sächs. schnirling, schnittling, dt. Schnittlauch; Taghete (Tagetes spp.) < sächs./dt. Tagetes bzw. aus der wissenschaftlichen Benennung dieser Zierpflanze nach dem Namen des Halbgottes Tages; Tुelăr, țeler, ţeleră (Apium graveolens) < sächs. zellər, dt. Zeller; țucroş, țucuran, țucurechi (Beta vulgaris var. altissima), Zuckerrübe, sächs. zackerriep, ung. cukorrépa, cukrosrépa.

(4) Andere Wortformen fremden Ursprungs: abricoji, abricoz (,Aprikose'; die Formen abricoji, abricoz, durch die Stimmhaftigkeit der Konsonant $p$ erhalten, sind mit fr. abricot, abricot-pêche und abricotier "Aprikosenbaum", span. albaricoque, port. albricoque zu vergleichen, alle vom arab. al barquq (al birquq) ,frühreif', vgl. lat. praecoqum malum ,frühreifer Apfelbaum' und lat. apricus ,sonnenbescheint; reif'; carfiol, cartifiol, cartofiol, cartofiom (Brassica oleracea var. botrys) < dt. Karfiol < ital. dial. cavol fior, sächs. kardiviol(ən), kardiwiol > ung. kárfiol, kartifiol, skr. karfijol, ukr. karafiol; cartoafe (Dahlia cultorum, Solanum tuberosum) die erste Pflanze hat in der Erde knollige Wurzeln, die zweite Knollen $<$ dt. Kartoffel $<$ it. tartufoli, tartiffolo "Trüffeln”, weil die Knollen diesen unterirdischen Pilzen ähnlich ausehen; castan (Aesculus hyppocastanum, Catanea sativa) < dt. Kastanienbaum, paleoslav. kastanヶ, bulg. kástan, kesten, wissenschaftliche Benennung castanea > ngriech. kástanon ,Kastanie' in Verbindung mit altind. kashta- ,Wald'; cocarde, coarde (Gaillardia picta) < dt. Kokardenblume < fr. cocarde; creţăruş(i) (Bellis perennis) < cre(i)ţar, crăiţar ,Kreuzer' 
(Münze) < dt. Kreuzer, ung. krajcár; fenicel (Foeniculum vulgare); fiache (Viola odorata); lilie (Iris florentina, Iris germanica, Lilium bulbiferum, Lilium candidum, Lilium martagon), lilioane (Lilium candidum), liliom (Hemerocallis fulva, Lilium candidum), lilion (Hemerocallis fulva, Iris germanica, Lilium candidum), liliu (Lilium candidum), liliuăn (Iris germanica), liliuţă (Anthericum ramosum), lilom (Iris spp.) < dt. Lilie, sächs. Leljen < lat. lilium ,Lilie', verbreitet auch unter dem Beinamen liliom, lilion, unter ungarischem Einfluss der Benennung liliom, alle Pflanzen sind Lilienarten oder damit verwandt; Maieran, maioran, maiorean, măierean( $\breve{a})$, maiorană, măgheran (Majorana hortensis, Origanum vulgare), măgherean (Majorana hortensis), măghieran, măghiran, măghieran, măgieran, măgiran (Majorana hortensis), măioreană (Majorana hortensis) < dt./sächs. Majoran, Meieran, poln. und skr. majeran, russ. maioran, skr. majoran, ung. Majorán, alle $<$ wissenschaftliche Benennung majorana, arabischer Herkunft, oder aus lat. majorana, welches auf lat. major (größer, kräftiger') zurückgeht oder von Dioscorides erwähnten altlat. meyurana bzw. weil es sich um eine bevorzugte aromatische Pflanze handelt, mit dem mgriech. mágeiros, ngriech. mageirion ,Küche' zu vergleichen; Pizang (Musa spp.) < dt. Pisang < pisang ist der Name der Banane bei den Malaysiern und Indonesiern; porobici, porodăi, porodăici, porodeici, porodică, porodici, porodiciuri, porodiţă (Lycopersicon esculentum) < ung. paradicsom < dt. Paradeisen; rabarbară (Rheum officinale), rabarbură (Rheum officinale, Rheum palmatum), rabarbăr (Rheum rhabarbarum, Rheum rhaponticum), rebarbar( $\breve{a})$ (Rheum officinale, Rheum palmatum, Rheum rhaponticum), rebarboră, robarbă, robarbăr (Rheum palmatum) < sächs. rabarbər, dt. $\mathrm{R}(\mathrm{h})$ abarber, it. rabarbaro < rhabarbarum, radix barbarum (,Barbarenwurzel'); ramie (Boehmeria nivea) $<\mathrm{dt}$. Ramie, fr., engl. ramie $<$ malaysisch rameh; strălucită (Gypsophila paniculata) aus dem rum. Verb a străluci (Präfix stră- und „,a luci” < lat. lucere), (aus)strahlen', oder Kombination zwischen i.-e. stra-, vgl. dt. Strahl und das rum. Verb a luci, die Pflanze hat viele, reiche, weiße oder rötliche Blüten.

(5) Lehnprägungen sind Benennungen, die unter Verwendung von sprachlichen Mitteln der Empfängersprache zustandekommen sind. Hierbei handelt es sich um eine Nachbildung eines fremdsprachlichen Inhalts mit den Mitteln der Empfängersprache (hier: des Rumänischen). Dabei kann die Adaption der Übernahmen auf verschiedene Weise erfolgen:

(a) Lehnbedeutung: Die Anpassung geschieht durch Wandel bzw. Erweiterung der Bedeutung heimischer Wörter, d.h. es geht um eine Bedeutung, die ein Wort unter fremdsprachlichem Einfluss annimmt, wodurch eine Umdeutung der ursprünglichen Bedeutung bzw. eine Bedeutungserweiterung stattfindet. Bei einer Lehnbedeutung wird nur die Bedeutung und nicht das Wort übernommen und auf ein einheimisches Wort übertragen. Lehnbedeutungen entstehen oft durch Interferenzen bei Sprachträgern, durch den hohen Verwandtschaftsgrad zwischen den Sprachen. Auffal- 
lend ist hierbei, dass Bedeutungserweiterungen von Lexemen einer Sprache nach dem Vorbild einer anderen Sprache nicht nur auf der Klangähnlichkeit beruhen, sondern auch die inhaltsbezogene Teilidentität zwischen Lexemen der beiden Sprachen berücksichtigen: coif (Aconitum spp.) $<\mathrm{dt}$. Eisenhut, rum. coif ,Helm' < lat. cofea; weist auf die Blütenform hin; focul drăguţei (Lychnis calcedonica) < dt. brennende Liebe; rum. foc ,Feuer' $<$ lat. focus, rum. drăguţei ist die Genitivform von rum. drăguţă ,Geliebte ${ }^{\prime}<$ drag $<$ slaw. dragǔ; die Pflanze hat rote Blüten.

(b) Lehnübersetzung: Bezeichnet das Ergebnis einer genauen Glied-fürGlied wiedergebenden Übersetzung eines fremdsprachlichen Vorbildes. Dabei wird auch den identischen/ähnlichen und semantischen äquivalenten Wortbildungsmustern Rechnung getragen: arbore/copac de ambră (Liquidambar orientalis) < dt. Amberbaum; arborele de bumbac (Bombax ceiba) $<\mathrm{dt}$. Baumwollbaum; arbore de chinină (Cinchona spp.) Chininbaum; arbore de gumă (Ficus elastica) < dt. Gummibaum; arbore de mastic (Pistacia lentiscus) < $\mathrm{dt}$. Mastixstrauch; arborele de pâine al maimuţelor (Adansonia digitata) $<\mathrm{dt}$. Affenbrotbaum; arborele mamut (Sequoia gigantea; heute: Sequoiadendron giganteum) < dt. Mammutbaum; aripa ingerului (Caladium bicolor) $<\mathrm{dt}$. Engelsflügel; castan galben (Aesculus octandra, heute Aesculus flava) $<\mathrm{dt}$. gelber Kastanienbaum, castan indian (Aesculus indica) < dt. indischer Kastanienbaum oder von der wissenschaftlichen Benennung, castan japonez (Aesculus turbinata) < dt. japanischer Kastanienbaum, castan roşu (Aesculus carnea) $<\mathrm{dt}$. roter Kastanienbaum; căcatul dracului (Ferula asa-foetida) $<\mathrm{dt}$. Teufelsdreck, die Pflanze hat einen unangenehmen Geruch (vgl. auch die wissenschaftliche Bennenung foetida ,stinkend'), das rum. Hauptwort căcat (,fecale') < lat. cacatus (könnte auch vorlateinisch sein); cânepă de Manila (Musa textilis) < dt. Manilahanf; coada peştelui, coadă de peşte (Caryota maxima $<$ dt. Fischschwanzpalme, weist auf die Form der Blätter hin; copacul furnicilor (Cecropia palmata) < dt. Ameisenbaum; copacul lanternă (Crinodendron hookerianum) $<\mathrm{dt}$. Laternenbaum, die Blumen sehen wie Laternen aus; copacul leopard (Caesalpinia ferrea) $<\mathrm{dt}$. Leopardenbaum, die Rinde ähnelt dem Leopardenfell; copacul popii (Evonymus europaeus) < möglicherweise dt. Pfaffenholz, die Früchte werden von den Rumänen Pfarrerkappe genannt, weil sie der Kopfbedeckung der Priester ähnlich sind; auch die Sachsen nennen sie Pfaffenkäppchen; coroana împăratului (Fritillaria imperialis, Lilium bulbiferum), coroană împărătească (Fritillaria imperialis) < dt. Kaiserkrone, rum. coroană ,Krone, Kranz' < lat. corona; die Blüten sehen so aus; crinul focului (Hemerocallis fulva) < dt. Feuerlilie; rum. crin soll vom slaw. krinŭ abstammen; vgl. auch die wissenschaftliche Benennung crinum < griech. кpıvov, krinon ,Lilie', rum. foc < lat focus; două foi (Listera ovata) $<\mathrm{dt}$. Zweiblatt; die Pflanze hat zwei Blätter; rum. foi < lat. folia; fasole calabar (Physostigma venenosum) $<\mathrm{dt}$. Kalabarbohne nach der gleichnamigen Küste in Westafrika, wovon diese Art abstammt; fasole-soia (Glycine hispida) $<\mathrm{dt}$. Sojabohne aus ngriech. fasóli und lat. phaseolus, beide aus griech. pháse- 
los ,Boot', auf die Form der Früchte hinweisend; fenicul de mare (Chrithmum maritimum) < dt. Meerfenchel, der erste Teil der wissenschaftlichen Benennung lat. foeniculum, möglich auch unter dem Einfluss des sächs. fienchəl, dt. Fenchel; feriga-sabie (Nephrolepis sp.) < dt. Schwertfarn; ferigea vărgată (Asplenium trichomanes) Streifenfarn, das zweite Wort aus dem lat. variegata; ferigă solzoasă (Notholaena maranthae heute Cheilanthes maranthae) $<\mathrm{dt}$. Schuppenfarn, solz vgl. lat. solidus ,Goldmünze'; floarea broaştei, floarea broaştii, floarea broaştelor (Taraxacum officinale) < dt. Krötenblume, die Pflanze wächst an feuchten Orten; rum. broască von lat. *brosca oder autochthon, da alb. breskë; floarea Domnului (Dodecatheon pulchellum) < dt. Gottesblume (dodecatheon bedeutet ,zwölf Götter', auf die wunderschönen vielen Blüten hinweisend); floarea flamingo (Anthurium spp.) < dt. Flamingoblume, die Pflanze hat Blüten, die dem Flamingovogel ähnlich sind (Phoenicopterus ruber); floarea gramofonului (Pharbitis purpurea) < dt. Grammophonblume, die Blumen haben die Form des Grammophontrichters; floarea lui Io(a)n (Hypericum perforatum), floarea lui Sf. Ion, floarea lui Sântion (Galium verum) Johannisblume, die Blüten der Pflanzen erreichen ihre volle Pracht am 24. Juni; in Rumänien wird Johannes der Täufer an diesem Tag gefeiert; floarea pasărea paradisului (Strelitzia spp.) Paradiesvogelblume, wegen ihrer Schönheit so benannt; fructul pasiunii (Passiflora edulis) Passionsfrucht; floarea Paştelui (Anemone nemorosa) < dt. Ost(er)blume, sächs. Uistərblom; frunza sângerie (Iresine herbstii) < dt. Blutblatt, frunză ,Blatt' vgl. lat. *frondia < lat. frons, -dis, rum. sângerie ,blutartig' < sânge "Blut'; vgl. lat.*sanguem < sanguis; ghimber nemţesc (Arum maculatum) < dt. deutscher Ingwer, da die Knolle der Wurzel dem Ingwer (Zingiber officinale) ähnlich ist; grâul vacii (Melampyrum pratense, M. sylvaticum) < dt. Kuhweizen, die Kühe, rum. vaca vgl. lat. vacca, altind. vaçā, fressen die jungen Pflanzen, die Butter bekommt eine gelbe Farbe und ist geschmackvoller; hribul cizmarului (Boletus luridus) < dt. Schusterpilz (Boletus erythropus), rum. hrib "Pilz" < ukr. hryb, tschech., slow. hřib (Boletus), russ. grib(î) ,Pilz', das Hauptwort cizmar ,Schuster' < cizmă < ung. csizma, tc. çizme; iarba buricului (Umbilicus sp.) < dt. Nabelkraut; rum. buric < lat. *umbulucus < lat. ummbilicus; rum. iarbă ,Gras, Kraut' < lat. herba; iarba ficatului (Hepatica nobilis) < dt. Leberkraut, aus der wissenschaftlichen Benennung hepatica abgeleitet; iarba inimii (Leonurus cardiaca) < dt. Herzkraut, Herzgespann, von der wissenschaftlichen Benennung cardiaca beeinflusst; iarba păretelui (Parietaria officinalis) < $\mathrm{dt}$. Mauerraute, lat. parietaria; iarba piperiului (Satureja hortensis) $<\mathrm{dt}$. Pfefferkraut; iarbă arzătoare (Clematis recta) $<\mathrm{dt}$. Brennkraut, a arde ,brennen' lat. ardere; iarbă de cositor (Equisetum spp.) < dt. Zinnkraut (die Stiele enthalten Kieselsäure und werden zum Polieren von Zinngegenständen benutzt); iarbă de molii (Verbascum blattaria) < dt. Mottenkraut, Schabenkraut, lat. blatta ,Motte', die Staubblätter sehen aus wie Mottenantennen; iasomia nopţii (Cestrum nocturnum) < dt. Nachtjasmin, von der wissenschaftlichen Benennung Jasminum, ngriech. ghiasemi, iasemi < pers. 
yasmine abstammend, die Pflanze sieht dem Jasmin (Jasminum) ähnlich und der Geruch ist in der Nacht stärker, rum. noapte; vgl. lat. nox, noctis; isop de apă (Bacopa myryophylloides) < dt. Wasserysop, sieht dem Ysop (Hyssopus officinalis) ähnlich und wächst im Wasser; rum. apă vgl. lat. aqua oder i.-e. Wurzel *ap- „apă"; lacrima miresii (Briza media) < dt. Brautzähre ,Brautträne'; die Pflanze hat Ähren, die wie Tropfen oder Tränen aussehen; lapte de vrăjitoare (Chelidonium majus) < dt. Hexenmilch, wegen dem Latex der Pflanze so benannt; laur de munte (Umbellularia californica) $<\mathrm{dt}$. Berglorbeer, rum. laur < lat. laurus (Laurus nobilis), ist dem Lorbeer ähnlich aber nicht verwandt; leuştean negru (Smyrnium olusatrum) < dt. schwarzer Liebstöckel, das deutsche Phytonym selbst ist eine Lehnübersetzung < lat. levisticum < griech. libystikon ,(Pflanze) aus Ligurien', das rum. Adj. negru ,schwarz' < lat. nigrum; Lizuca cea harnică (Impatiens sultani) < dt. Fleißiges Lieschen, eine Zierpflanze mit reichen Blüten; lucernă de Banat (Medicago sativa) < dt. Banater Luzerne (im Banat bevorzugt); maţele găinilor (Stellaria media) < dt. Hühnerdarm, rum. maţ ,Darm' < lat. matia, găină ,Huhn, Henne' < lat. gallina; die Pflanzenbenennung weist auf die Form des Stiels hin; mazăre ţucără (Pisum sativum) < dt. Zuckerbohne; Russu (1981) legt das rum. Phytonym mazăre ,Erbse, Bohne' als autochthon aus, rum. ţucără < dt. Zucker, vgl. arab. sukkar ,Zucker'; măcriş de mare $($ Nasturtium officinale) $<\mathrm{dt}$. Meerampfer, rum. mare ,Meer' < lat. mare; die Pflanze wächst eigentlich nicht am Meer, sondern neben Bächen und Quellen; paradis (Malus pumila var. paradisiaca) < dt. Paradies-Apfel; măr de balsam (Clusia major) < dt. Balsamapfel, engl. balsam apple; pară de pământ (Lathyrus tuberosus) < dt. Erdbirnchen, hat birnförmige knollige Wurzeln; pintenul cocoşului (Crataegus crus-galli, Plectranthus fruticosus) < dt. Hahnensporn < wissenschaftliche Benennung plectranthus < griech. plektron ,Sporn' und ánthos ,Blume'; piperul călugărilor (Vitex agnus-castus) $<\mathrm{dt}$. Mönchspfeffer, die Früchte haben einen brennenden, scharfen Geschmack und wurden besonders von Mönchen anstatt Pfeffer verwendet, rum. piper ,Pfeffer' < (n)griech. pipéri, slaw. piperŭ, piperĭ $<$ pers. pippari $<$ altind. pippali; plăcintăblam (Callistephus chinensis) < sächs. plətschintəblâm, dt. Palatschinkenblume; Verbindung zwischen rum. plăcintă ,Palatschinken, Strudel' und dt. Blume; rum. plăcintă < lat. placenta, weist auf die Blütenform hin; ploaie de aur (Forsithia suspensa, Laburnum anagyroides) $<\mathrm{dt}$. Goldregen, die Pflanze hat goldgelbe Blumen wie große Regentropfen; rum. ploaie ,Regen' < lat. *plovia < lat. pluvia; pom de mandulă (Amygdalus communis actualmente Prunus dulcis) < dt. Mandelbaum, rum. pom ,Baum' $<$ lat. pomus; punguliţă de stânci (Aethionema saxatilis) < dt. Steintäschel, die Pflanze hat taschenförmige Früchte; rum. pungă ,Tasche', rum. punguţă ,Täschchen'; vgl. ngriech. ponga/pongi, lat. med. punga, slaw. pangva, gotisch. Pugg, rum. stâncă ,Felsen' ist autochthon; putelizi, pute Lizi (Pelargonium zonale) < dt./sächs. Stinkende Liesel/Lieschen, Liese; die Pflanze hat einen unangenehmen Geruch; rădăcina ciumei (Petasites albus, Petasites 
hybridus) < dt. Pestwurz; rădăcina sângelui (Potentilla erecta) < dt. Blutwurz; die Wurzeln sind rötlich und werden zur Herstellung einer roten Farbe benutzt, die Pflanze wurde auch zur Blutstillung verwendet; rum. sânge ,Blut'; vgl. lat. *sanguem < sanguis; rădăcina şerpilor (Polygonum bistorta, Polygonum viviparum) $<\mathrm{dt}$. Schlangenwurz (Dracunculus vulgaris), Natterwurz, vgl. lat. herba serpentina; die Wurzelstöcke sehen den Schlangen ähnlich aus; rum. şarpe 'Schlange' < lat. serpes < lat. serpens, kann aber auch vorlateinisch sein; die Staubblätter sehen wie Schlangenzungen aus; rădăcina ursului (Ligusticum mutellioides) < dt. Bärwurz; rădăcină de sânge (Sanguinaria sp.) < dt. Blutwurzel; rum. sânge ,Blut' vgl. lat. *sanguem < sanguis; regele moliilor (Plectranthus fruticosus) $<\mathrm{dt}$. Mottenkönig, giftig für Insekten; rută de grădină (Ruta graveolens) < dt. Gartenraute/Rute < griech. rhutos ,behütet'; salată de apă (Pistia stratiotes) < dt. Wassersalat, rum. apă < lat. aqua, i.-e. Wurzel *ap- ,Wasser'; scoarţă de friguri (Cinchona officinalis) < dt. Fieberrindenbaum; die Rinde wird als Heilmittel für Malaria verwendet; smalt de sticlă (Salicornia europaea) < dt. Glasschmeltz; die Pflanze sieht aus, als wäre sie aus Glas; rum. sticlă ,Glas' < slaw. stǐklo; spanac englezesc (Rumex patientia) < dt. Englischer Spinat < ngriech. spanáki < pers. aspanakh; spinul crucii (Rhamnus cathartica) < dt. Kreuzdorn; entweder weil die Dornen mit dem Ast ein Kreuz bilden, oder weil Jesus Krone vor der Kreuzigung aus dieser Pflanze gewesen sein soll; rum. spin 'Dorn' < lat. spinus; struţa apei (Veronica anagallis-aquatica), struţa mirelui, struţul mirelui (Erigeron canadensis), struţişori (Selaginella kraussiana, Selaginella selaginoides), struţuşor (Lycopodium selago heute Huperzia selago), struţuşori (Selaginella helvetica, Selaginella selaginoides) < dt. Strauß; auch im Rumänischen sowohl Blumenstrauß als auch der afrikanische Vogel; rum. struţişor ,Sträußlein' bedeutet sowohl ,Blumensträußlein' als auch ,Trauben'; trestie de Spania (Calamus caesius) < dt. Spanisches Rohr; die Pflanze stammt aber aus Malayesien und Kalimantan; trifoi persan (Trifolium resupinatum) < dt. Persischer Klee; tufă de foc (Hamelia petens) < dt. Feuerbusch, engl. firebush < rum. tufă ,Busch, Gebüsch' < lat. tufa; ein Strauch mit feuerroten Blüten; rum. foc ,Feuer' < lat. focus; umbra nopții (Solanum dulcamara, Solanum nigrum) < dt. Nachtschatten (Solanum nigrum), lat. umbra noctis; die Pflanze ist dunkelgrün und hat schwarze Früchte; urzică surdă (Lamium album) < dt. Taubnessel; rum. surdă , taub' < lat. surda, rum. urzică $<$ lat urtica; verdeaţa iernii, verdiaţa iernii (Chimaphila umbellata, Pyrola media) < dt. Wintergrün.

(c) Lehnübertragung: Im Unterschied zur Glied-für-Glied-Wiedergabe der Lehnübersetzung basiert die Lehnübertragung auf einem freieren Umgang mit dem fremdsprachlichen Ausgangswort, das durch eine angenäherte Übersetzung oder aber genauere Ausdeutung wiedergegeben wird. D.h. es geht hier um Teilübersetzungen, freiere Übertragungen von Wörtern einer fremden Sprache: arbore de friguri (Cinchona pubescens) $<\mathrm{dt}$. Fieberrindenbaum; cănişoară (Nepenthes spp.), cănişoară purpurie (Sarracenia 
purpurea): Karnivorpflanze mit kannenförmigen Blüten als Falle für Insekten; rum. Hauptwort cană ,Tasse, Kanne', scheint vorlateinisch zu sein; die rumänischen Pflanzenbenennungen sind Lehnübertragungen des dt. Kannenpflanze; căpşună grasă (Fragaria moschata) < dt. Riesenerdbeere, rum. căpşună ,Erdbeere' < *căpşon < rom. căpşor < cap ,Kopf, Haupt', rum. grasă ,dick, fett' < lat. *grassus < lat. crassus, hier in der Bedeutung ,groß'; guleraş (Carpesium cernuum) < dt. Kragenblume, rum. guleraş ist die Diminutivform von rum. guler ,Kragen' < ung. gallér, weist auf die Blüten hin; floarea lui Cristos (Helleborus niger) < dt. Christrose, Weihnachtsrose; o mie de florini (Centaurium erythraea) < dt. Tausendgüldenkraut; weist darauf hin, wie wertvoll diese Heilpflanze war; finhienul bălţii (Oenanthe aquatica) $<\mathrm{dt}$. Wasserfenchel; die Pflanze sieht Foeniculum vulgare ähnlich, wächst aber in Teichen; floare de o zi (Commelina communis) < dt. Tagblume, rum. $z i$,Tag' aus dem lat. die; floare popească (Narcissus poëticus) < dt. pfarrersche Narzisse, sächs. fuaresch narziss, rum. popească < popă < slaw. popŭ, lat. popa; iarba miresii (Briza media) < dt. Brautzähre ,Brautträne', rum. iarbă < lat. herba; iarbă de doctorit ficatul (Ageratum haustonianum) $<$ dt. Leberbalsam aus lat. med. doctor und lat. ficatum; iarbă englezească (Lolium perenne) $<\mathrm{dt}$. Englisches Raygras, Englisches Weidelgras; lacrima doamnei (Ophrys fuciflora) < dt. (Hummel) Frauentränen, rum. lacrimă < lat. lacrima; lacrimile miresei (Convallaria majalis, Dicentra spectabilis) < dt. Brautzähre, sächs. brautzäir; rum. lacrimile ist die artikulierte Pluralform des rum. lacrima < lat. lacrima; lemnul Sanctului Ioan (Ribes rubrum) < dt. Johannisbeerstrauch; păr de stâncă (Amelanchier ovalis) < dt. Felsenbirne, rum. păr < lat. pirus/pyrus ,Birnbaum'; pomul Domnului (Ailanthus altissima) < dt. Götterbaum; eine Lehnübertragung von moluc ailanto (,Himmelsbaum').

(d) Semantische Veränderungen. Volksetymologie: barba Saftei (Rheum officinale, Rheum palmatum) < dt. Rhabarberschaft, das rum. Phytonym lautet ,Saftas Bart'; Safta ist ein weibliches Anthroponym; clei(u) (Medicago sativa, Trifolium spp.) < dt. Klee (Trifolium), Schneckenklee (Medicago); Clocăboambă (Aquilegia vulgaris) < sächs. kliokəbläom, klokəblommən, dt. Glocke(n)blume, rum. clocă ,Glucke, Bruthenne' und rum. boambă ,Weinbeere, Beere, Samen'; crinul fânului < dt. Feuerlilie, das rum. Phytonym crin soll von slaw. krinǔ abstammen, aber es gibt auch die wissenschaftliche Benennung crinum < griech. крıvov, krinon ,Lilie'; drăgan (Astragalus gummifer) < dt. (Gummi)Tragant, Dragant; floarea oştilor (Anemone nemorosa) < dt. Ost(er)blume, sächs. Uistərblom; franţ (Galinsoga parviflora) $<\mathrm{dt}$. Franzosenkraut; frăţiori (Viola tricolor). Dies sei nach Drăgulescu (2010) eine Eindeutung nach dt. Fratze; măzăriche (Briza media, Nigella arvensis) < sächs./dt. Maschehirsch < dt. Mönschenhirse (Briza media) bzw. sächs. muasertcher (Nigella damascena); măzăraş (Aposeris foetida) < sächs. Huasngras $<\mathrm{dt}$. Hasengras oder Muasergras; mierluţă (Minuartia verna) hat nichts mit dem rum. mierlă ,Amsel' zu tun; es handelt sich hier um eine Volksethymologie von *mieruţă nach dem dt./sächs. Miere; moş gras (Phalaris 
arundinacea var. picta) < sächs. moschəngras, dt. Maschengras; salată (Allium ascalonicum) < dt. Schallote, Schalloten; săgeţel (Carex arenaria heute Carex colchida, Gladiolus gandavensis) < dt. Segge, Segese ,Sense'; die Pflanzen haben schneidende Blätter < rum. săgeată ,Pfeil' < lat. sagitta; troacă (Cucurbita pepo, Lagenaria siceraria), trochiţe (Lagenaria siceraria) halbe Früchtenschalen wurden als kleine Troge benutzt, rum. Hauptwort troacă , Trog' $^{\prime}<$ sächs. troch, dt. Trog.

(e) Mischbildungen: hier handelt es sich um direkte phonetisch unveränderte Übernahmen aus der deutschen Sprache oder aus dem siebenbürgisch-sächsischen Dialekt, eine angenäherte Übersetzung oder Ausdeutung und ein rumänisches (sogar autochthones) Wort: acacie boierească $($ Sophora japonica) < dt. Akazie, rum. boierească < Adjektivalform des rum. boier < slaw. bolijarinu ,Herr'; bostan de lompău (Lagenaria siceraria): Kürbis, die Frucht wird dazu verwendet, sogenannte "lompău/lampă" fertigzustellen < dt. Lampe, rum. bostan < tc. bostan kabaği ,Gartenkürbis'; buşteanu(l) Ielelor: vgl. sächs. bumstam, dt. Baumstamm und rum. iele ,Feen/Hexen'; cârligei de somot (Viola wittrockiana) die Blüten sind samtig, rum. somot, somet 'Samt' < sächs. sumət, dt. Sammet, Samt, rum. cârlig „Hacken'; nach Russu (1981) auf das i.-e. Wurzel *(s)ker- ,zurückkehren, beugen, biegen' zurückführbar; chel varză creață (Brassica oleracea var. sabauda) < sächs. ki(e)l, kîl, dt. Köhl(kraut); cherbăl auriu (Chaerophyllum aureum), cherbăl sălbatic, chervăl sălbatic (Anthriscus sylvestris) < dt. Kälber(kropf), dt. Kerbel, sächs. kiərwəl, kerwəl, Wald/Wiessekerbel, < cerefolium < griech. chairophyllon , angenehmes Blatt'; chifăr roşu (Pinus sylvestris), chifer negru (Pinus nigra) < dt. Kiefer; corfa jupânesii (Tropaeolum majus) < rum. corfă ,Korb' < sächs. korf, dt. Korb > ung. karfa, es gibt auch ein griech. korban und lat. corbis davon fr. corbeille, rum. jupâneasă ,Bojarin; Frau aus der Stadt' $<$ Femininform von rum. jupân < slaw. županŭ; so genannt wegen der korbförmigen Blumen; crumpei de lună (Solanum tuberosum), crumpene porceşti, crumpi porceşti, crumpene sălbatice (Helianthus tuberosus) < sächs. krumpirn, dt. Grundbirne, unter dem Einfluss des ung. kolompér, kolompéra, krompé, krumpi, krumpli < dt. Grundbirne; feldera pământului, ferdela pământului (Cyathus spp.) rum. felderă, ferdelă ,Maßeinheit für Getreide' < sächs. fyrdəl, dt. Viertel ${ }^{18}$; floare din şanţ (?Sieglingia decumbens) wächst auf den Schanzen, ghinţură crăcoasă (Gentianella austriaca), ghinţură de primăvară (Gentiana verna), ghinţură galbenă (Gentiana lutea), ghinţură guşată (Gentiana utriculosa), ghinţură pătată (Gentiana punctata), ghințură tomnatică (Gentiana pneumonanthe), ghințură vânătă (Gentiana cruciata) < dt. Enzian; foalchin de iarnă (Cheiranthus cheiri), foaltine bogate (Mathiola incana ,mit reichen Blüten'), foaltine sărace (Mathiola incana , mit einfachen Blüten') < sächs. foaltchər, vâltchər, foalen $<\mathrm{dt}$. Veilchen (Mathiola incana), sächs. giel foalen, dt. gelbes Veilchen (Cheiranthus cheiri), sächs. voâlchen, dt. Veilchen (Viola spp.); grâu spelt (Triticum spelta) < dt. Spelt, Spelz, vgl. i.-e. Wurzel *pel-, pel-t- ,Staub, Mehl'; iarbă de 
tripăl (Digitalis grandiflora) zur Behandlung von Tripper verwendet; inţură galbenă (Gentiana lutea, Gentiana punctata) < dt. Enzian; levcoaie galbenă (Cheiranthus cheiri), levcoaie roşie (Matthiola incana) < dt. Levkoje $<$ altgriech. leucoion (Matthiola incana), aus leucós und ion gebildet, mit der Bedeutung ,weiße Veilchen'; es gibt aber auch Arten mit gelben (rum. galben) und roten (rum. roşu) Blumen; lilie albă (Lilium candidum), lilie galbenă (Iris pseudacorus, Lilium bulbiferum), lilie roşie (Lilium bulbiferum), lilie sălbatică (Iris germanica), lilie vânătă (Iris florentina, Iris germanica), liliom alb (Lilium candidum), lilion alb (Convallaria majalis, Lilium candidum), liliom roşu (Hemerocallis fulva), lilion albastru (Iris germanica), lilion bun (Convallaria majalis), lilion galben (Hemerocallis fulva), lilion păsăresc (Iris variegata), lilion sălbatic (Majanthemum bifolium), lilion vânăt (Iris germanica), liliu galben (Iris pseudacorus), liliu vânăt (Iris germanica) < dt. Lilie, sächs. Leljen < lat. lilium ,Lilie'; lipscănoaice, lipscănoaie (Coreopsis tinctoria) < rum. Lipsca < Leipzig unter dem Einfluss des slaw. Lipskŭ; lurbău de toamnă (Phlox paniculata) < sächs. lührber(buhm), lu(i)rbən, lu(i)rbər, liurbər, dt. Lorbeer, hat dem Flieder (Syringa vulgaris) ähnliche Blumen bis spät im Herbst; rum. toamna < lat. autumnus; mierluţă pitică (Minuartia recurva, Minuartia sedoides), mierluţă ţepoasă (Minuartia setacea); măghiranul pământului (Lysimachia nummularia) ist Majorana hortensis nicht ähnlich (einziges gemeinsames Merkmal sind die runden Blätter); der Name măghiranul pământului ,Erdmajoran' weist darauf hin, dass die Art wild ist; mihlele (Lycopersicon esculentum): nach der Ortschaft Mihla in Deutschland, woher diese Tomaten nach Rumänien eingeführt worden sind, vgl. das Anthroponym Mihle; ortul popii (Lysimachia nummularia) < rum. ort ,Münze' < dt. Ort, poln. ort, rum. popă < slaw. popǔ; die Blätter sehen wie kleine Münzen aus, vgl. die wissenschaftliche Benennung nummularia < lat. nummulus, wenig Geld, oder von geringem Wert'; părădăici roşii < sächs. Parədeis, dt. Paradeis(apfel), Paradies(apfel), ung. paradicsom < dt. Paradeisen; rosmalin cu miros mândru şi frumos (Philadelphus coronarius) < dt. Rosmarin; rabarbăr sălbatic (Polygonum cuspidatum) ist Rheum rhabarbarum ähnlich; rozmarin de baltă (Ledum palustre), rozmarin de munte (Gnaphalium silvaticum), rozmarinul câmpului (Ajuga chamaepytis) manche werden angebaut, andere wachsen wild auf Feldern, im Gebirge oder in den Mooren; spargă aninată (Asparagus sprengeri) < sächs. spargəl, dt. Spargel > ung. spárga, rum. Verb a anina ,(auf)hängen'; ţâtron de friguri (Sempervivum tectorum), ţâtron domnesc (Veratrum nigrum), țâtron sălbatic (Hedera helix, Ruscus hypoglossum), ţitroană, ţitrom (Citrus limon), ţitron (Citrus limon, Philadelphus coronarius) $<$ dt. Zitrone, Zitron, im Süden Siebenbürgens ist mit zitron eine immergrüne Pflanze gemeint; ţelăr sălbatic (Peucedanum sp., wahrscheinlich Peucedanum oreoselinum) < sächs. zellər, dt. Zeller, rum. sălbatic , wild' < lat. silvaticus , aus dem Wald, waldig' < lat. *salvaticus; urzică de şură (Urtica urens) < sächs. schyren, dt. Scheuer, Schur, vgl. ukr. šura, rum. urzică ,Nessel'< lat. urtica. 
Die Transferenzen können nach dem Ausmaß ihrer Integration in das rumänische Sprachsystem (Assimilation, Eingliederung, „Rumänisierung”) gegliedert werden in: (a) phonemische Integration: acacie, acaţie; bambus; bacolţi; berten, bertin; bonă; bordă; boreț; bufă, bufoi, bufon(iu); buhaci, buhaş, buhăşer; calarabă, calarambă, călărabe, caralabă, cararabe, cărălabă, chelărabe, chelarabă, chelărabi, chelărabie; carfiol; cârneauă, hernave; cartifiol, cartofiol, cartofiom; cartoafe; castan; chel, chil, chiel, chil; chelărade, chelerab; cherbel, cherbăl, chervăl; chifăr, chifer; clocăboambă; cocarde; crețăruş(i); cricin, crihim, crihin, crichin; crumciri, crumpe, crumpei, crumpene, crumpeni, crumperi, crumpi, crumpiri, crumple, crumpli, crupre; crumpei; cule; cuțcrişoară, cuțcrişor; diptam; dost; edălvais; ențură, enzură ; eră erdăpane; fefărminţ; fenicel; fenicel; fetică; fiache; fidireie; finc(h)en, finchăn, fenhiel, finhien, finchil, finc(h) in, fincăl; flos; foalcer, foalchine, foalâne, foaline, foaltăr, foalter, foaltine, foahine, foanchine; garva, garvă; gerbera; ghinţiure, ghinţură, ghinţurea, ghinţurele, ghinţurică; gonele; grap; grubă, grube, grumbă(i), grumbănă, grumbe, grumcizi, grumpă, grumpe, grumpene, grumpeni, grumpi, grumpin(i); hăidănuş(ă); heber; heber; heciumpeci, heciunpeci; holăr; holăr; hulcă; inghe, inguţe; ințură, ințurea, inţurică; levcoaie; libistoc; lilie; liurbă $(u), l(i)$ urben, l(i)urber, liorbe; liurben, lurbău; liză; mace; maieran, maioran, maiorean, măierean( $\breve{a})$, maiorană, măgheran, măgherean, măghieran, măghiran, măghieran, măgieran, măgiran, măioreană; mandulă; mangold; minuţe; mure; mutmel, mutruel, muturel; nudli; ortanță, ortensă, ortensie, ortenzie; părădaice, părădaisăne, părădaise, părădăi, părădaişi, părădazine, părădăici, parad(a)ise, paradaice, paradaise, paradice, paradici, bărădăi, porobici, porodăi, porodăici, porodeici,

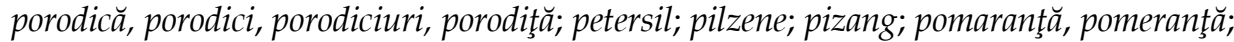
pospanc, pospang, puspan, puşpan, sospa(i)n; rabarbară, rabarbură, rabarbăr, rebarbar( $\breve{a})$, rebarboră, robarbă, robarbăr; ramie; râpac, râpă, râpe, rips; rapăn; rebizlă, ribiză, ribizil, ribizle, ribizli, ribizlă, ribuţe, răbiză, ribizle; rosmailin, rosmalin, rosmarin, rosmarint, rosmălin, rosmărin, rosmolin, rozmalin, rozmarin, rozmolin; ruben; şalotă; şarlai, şerlai, şerlau; silairei; simsă; şlaierblume; şnirling; soloagăr; spargă; şpic; spichinat, spichinel; spinac; spinat; ştainglezăr; strelitiia; taghete; țâncraus; ţâtroană, țâtron, ţitroană, ţitrom, ţitron; ţelăr, ţeler, ţeleră; țicorie; țimat, ţimărt, ţimăt, ţimet, ţâmărt; titenchi, titenghi; toibă; triftărele; trimpăn, trinpăn; țucroș, țucuran, țucurechi; vasărlilie; vermut; zegras u.a.; (b) graphemische Integration: die rumänische Rechtschreibung gründet auf dem phonologischen Prinzip, sodass die oben aufgezählten Pflanzennamen genauso geschrieben wie sie ausgesprochen werden; (c) semantische Integration: Übernahmen mit einer von der Herkunftssprache teilweise abweichenden Bedeutung sind oft bemerkbar (vgl. die Beispiele oben); (d) sprachsoziologische Integration: Übernahmen mit einem geringen Integrationsgrad aufgrund des Vorkommens in der fachgebundenen Kommunikation und in exklusiven Kreisen (Eliten) kommen auch vor. Die meisten Formen werden von den Dorfbewohnern benutzt und sind, mit wenigen Ausnahmen, nur in Siebenbürgen belegt.

\section{4. $\quad$ Fazit}

Das Inventar an rumänischen Pflanzennamen ist auch durch die Übernahmen 
aus dem Deutschen und seiner Standardvarietät Österreichisches Deutsch sowie den deutschsprachigen Dialekten Siebenbürgens (das SiebenbürgischSächsische und das Landlerische) bereichert worden. Bei der Herleitung der im Wörterbuch aufgenommenen Bezeichnungen wurde ihr Herkunftsgebiet bzw. die Ortschaft berücksichtigt, um eventuelle Wortvarianten eruieren zu können, die keine Pflanzenbezeichnungen darstellen und folglich nicht zum Forschungsgegenstand gehören. Dabei sind als Vergleichsgrundlage oft Pflanzenbezeichnungen der Siebenbürger Sachsen herangezogen worden.

In meiner bisherigen wissenschaftlichen Tätigkeit bin ich auf die enge Beziehung zwischen Mensch und Pflanzenwelt eingegangen, wobei ich auch Quellen der (sekundären) Nomination, Denotation und Konnotation erfasst habe. Mit dem hier lexikografisch umrissenen Bereich, der Ethnobotanik, tut sich ein weites Forschungsgebiet auf, das für anthropologische, psychologische oder psycholinguistische Untersuchungen verwendbar ist, da die Pflanzennamen ein linguistisch wertvoller Schatz darstellen, auf vielfältige Repräsentationen hinweisen und den affektiven Unterbewußtsein einer Gemeinschaft enthüllen.

\section{Endnoten}

1. Mehr über das Verhältnis zwischen Linguistik und Lexikographie siehe Hartmann (2015: 73-74).

2. Eine hervorragende Forschung hat Reichmann (2012) unternommen. Siehe dazu auch Smit (2016).

3. Mehr dazu siehe Drăgulescu (2010: 18).

4. Vgl. hierzu Lexiconul lui Mardarie Cozianu (1649) 42 Bezeichnungen, Codex Sturdzanus (ca. 1660-1670), Lexiconul Marsilian (ca. 1700) oder das Lexikon aus dem Fragmentul de la Bistriţa (ca. 1700) 75 Bezeichnungen, siehe Drăgulescu (2010: 18).

5. Vgl. Borza (1958) und (1961).

6. Vgl. das Kochbuch von Mihaiu (1749), Istoria poamelor (1773) oder die Arbeiten von Griselini (1780) und Raicevich (1788), mehr darüber in Drăgulescu (2010: 18).

7. Drăgulescu (2010: 18).

8. Zum Dicţionarul Borza vgl. Chivu (2008) und (2010 I: 333-340).

9. Drăgulescu (2010: 18).

10. Dies ist der Sammeltätigkeit zahlreicher Fachleute, Biologen, Ärzte, Volkskundler, Philologen, zu verdanken: hierzu ausführlicher Drăgulescu (2010: 18).

11. Es handelt sich hierbei um Bezeichnungen, die D. Brândză, J. Chr. Baumgarten, Fl. Porcius, G. Bariţiu, A. Fătu, N. Şuţu, I. Czihak, G. Crăiniceanu, I. Bob u.a. anführen.

12. So kam ein Inventar von über 1000 rumänischen Pflanzenbezeichnungen zustande.

13. Vgl. Drăgulescu (2010).

14. C. Drăgulescu (2010) meint, dass die Benennung nicht mit dem rum. Hauptwort „fată" (Mädchen) in Verbindung gebracht werden kann. Er verweist auf dt. fettig, auf die "fetten Blätter", die als Salat verzehrt wurden; möglich auch dt. Feldsalat.

15. Möglicherweise eine schwäbische Variante, da die Benennung im Kreis Caras-Severin verbreitet ist. Die Blätter sehen wie Nudeln aus.

16. 1773 wurde sie zu Ehren der britischen Königin Sophie Charlotte, einer geborenen Prinzessin von Mecklenburg-Strelitz und Gemahlin Königs Georg III., benannt. 
17. Vgl. awest. pairi-daeza (,mit Mauern umschlossener Ort', später ,Jagdpark, Jagddomäne') und auch die Bildungen mit para und diza (,umschlossener Ort, Burg') in zahlreichen trakischen Toponymen.

18. Diese Pilze werden auch von den Deutschen Erdviertel genannt.

\section{Literatur}

Borza, Al. 1958. Numiri româneşti de plante în vocabulare şi dicţionare din sec. XVII-XVIII. Cercetări lingvistice. Cluj, III.

Borza, Al. 1961. Aportul etnobotanic al lexicografiei vechi slavo-române şi al altor lucrări din secolul al XVIII-lea. Studii şi comunicări. Bucureşti, III und Apulum. Alba Iulia, IV.

Borza, Al. 1968. Diç̧ionar etnobotanic. Bucureşti: Academia R.S.R.

Chivu, Gh. 2008. Dictionarium Valachico-Latinum. Primul dicţionar al limbii române. Studiu introductiv, ediţie, indici şi glosar de Gh. Chivu. Bucureşti: Academia Română.

Chivu, Gh. 2010. Nume de plante în Dictionarium valachico-latinum. Limba română. Controverse, delimitări, noi ipoteze. Bucureşti: Universităţii din Bucureşti.

Drăgulescu, C. 2010. Diç̧ionarul explicativ al fitonimelor româneşti. Sibiu: Editura Universităţii „Lucian Blaga".

Drăgulescu, C. 2014. Dicţionar de fitonime româneşti. Sibiu: Editura Universităţii „Lucian Blaga”.

Hartmann, R.R.K. 2015. Lexicography and Its Interdisciplinary Contacts, with Special Reference to Linguistics and Onomasiology. Lexikos 15: 70-89.

Reichmann, O. 2012. Historische Lexikographie. Ideen, Verwirklichungen, Reflexionen an Beispielen des Deutschen, Niederländischen und Englischen. Studia Linguistica Germanica 111. Berlin/Boston: Walter de Gruyter.

Russu, I.I. 1981. Etnogeneza românilor. Bucureşti: Ştiinţifică şi Enciclopedică.

Smit, M. 2016. Oskar Reichmann. Historische Lexikographie. Ideen, Verwirklichungen, Reflexionen an Beispielen des Deutschen, Niederländischen und Englischen. Studia Linguistica Germanica 111. 2012, 586 pp. ISBN 978-3-11-028255-9. e-ISBN 978-3-11-028269-6. Berlin/Boston: Walter de Gruyter. Lexikos 26: 446-455. 